\title{
Diseño de sistema híbrido basado en energía solar PV con almacenamiento en baterías: aplicación a la enseñanza de Microrredes Eléctricas utilizando Microsoft Excel
}

\author{
Carlos Vargas-Salgado ${ }^{a}$, Paula Bastida Molina ${ }^{b}$, Lina Montuori ${ }^{\mathrm{c}}{ }^{\mathrm{d}}{ }^{\mathrm{J}}$ Jesús Águila-León \\ aDepartamento de Ingeniería Eléctrica. Camino de Vera, s/n Edificio 5E, Universitat Politècnica de \\ València carvarsa@upvnet.up.es \\ bInstituto Universitario de Ingeniería Energética, Camino de Vera s/n, Edificio 8E, 2a planta, \\ Universitat Politècnica de València paubasmo@etsid.upv.es \\ 'Departamento de Termodinámica Aplicada, Universitat Politècnica de València, Camino de Vera, s/n, \\ edificio 5J, 2 ${ }^{\text {a }}$ planta. 46022 Valencia (España), 1montuori@upvnet.upv.es. \\ dDepartamento de Estudios del Agua y la Energía, Universidad de Guadalajara \\ jesus.aguila@academicos.udg.mx
}

\begin{abstract}
In one of the laboratory practices of the electrical smart-grids course, the design of a microgrid (PV and battery system) is requested. Currently, to make thas kind of design there are software used by companies, such as the PVSyst for designing or Homer to carry out economic analysis. These tools, although useful, have a high cost and, as usual in computer programs, have limitations when it is required to modify the method used to carry out calculations. The design could also be carried out manually, but it is a very laborious procedure and mistakes can be made during the calculations. This document explains and uses a methodology to design a $12 \mathrm{~kW}$ power generation system through a viable tool used by students during their studies, such as Microsoft Excel. As a result, the design of the proposed system is obtained, which includes the selection and sizing of the solar panels, inverters, and batteries, as well as the integration of the components in the entire system.
\end{abstract}

Keywords: Photovoltaic system, storage system, microgrid, renewable energy, Teaching / learning methodologies.

\section{Resumen}

En una de las prácticas de la asignatura Redes eléctricas inteligentes del Máster Universitario en Tecnología Energética para Desarrollo Sostenible de la UPV, se pide realizar el diseño de una microrred con un sistema solar PV y almacenamiento en baterías. Actualmente para realizar dicho diseño existen programas informáticos utilizados a nivel empresarial, como el PVSyst o el SAM, para el caso del diseño o Homer para llevar a cabo el análisis económico. Estas herramientas, aunque útiles, tienen un coste elevado y, como es habitual en programas informáticos, tienen limitaciones a la hora de modificar el método a utilizar. Al introducir los cálculos en MS Excel por sí mismo, comparado con softwares, se mejora el aprendizaje de los estudiantes. También se podría realizar el diseño de forma manual, pero es un procedimiento muy laborioso y se pueden cometer errores durante la realización de 
los cálculos. En este documento se explica y utiliza una metodología innovadora para realizar el diseño de un sistema de generación de energía de $12 \mathrm{~kW}$, a través de una herramienta viable y utilizada por los estudiantes durante sus estudios como es el caso de Microsoft Excel. Como resultado de aplicar la innovación, el estudiante obtiene el diseño del sistema propuesto, el cual incluye la selección y el dimensionamiento de los paneles solares, inversores y baterías, así como la integración de todos los componentes en el sistema. Mediante este diseño el estudiante desarrolla las competencias transversales UPV 1 a 5 y 9.

Palabras clave: Solar PV, renovables, baterías, sistemas aislados, Metodologías de enseñanza/aprendizaje.

\section{Introducción}

Actualmente, dos de los sistemas más importantes en una microrred eléctrica aislada para autoconsumo son el sistema solar fotovoltaico y el sistema de almacenamiento de energía. La unidad didáctica 6 de la asignatura Redes eléctricas inteligentes del Máster Universitario en Tecnología Energética para Desarrollo Sostenible de la UPV, trata el tema de las microrredes, como se puede ver en su guía docente (Roldan, 2018). En la práctica 2 de la asignatura se diseña una microrred basada en energías renovables con almacenamiento en baterías. Este tema es tratado en muchas otras universidades principalmente en asignaturas de Master (Universidad de Valladolid, 2019) (Universitat Politécnica de Catalunya, 2019) (Universidad de Zaragoza, 2019), aunque también se imparte en menor medida en asignaturas de grado (Universidad de Almería).

Por otra parte, debido al aumento de la eficiencia y disminución del coste de los dispositivos más importantes de una instalación de autoconsumo (Paneles solares, baterías, inversor/cargador e inversor de conexión a red) y con la entrada en vigor del real decreto 244/2019, se prevé un aumento significativo de montajes de instalaciones solares fotovoltaicas, lo cual beneficiaría al sector (Energías Renovables, 2019). Por ello, es muy importante que los estudiantes se preparen para el diseño de instalaciones reales, de tal manera que puedan utilizar sus conocimientos a nivel profesional en función de la demanda del mercado.

Mediante esta metodología el estudiante desarrolla las competencias transversales CT-01 Comprensión e integración, CT-02 Aplicación y pensamiento práctico, CT-03 Análisis y resolución de problemas, CT-04 Innovación, creatividad y emprendimiento, CT-05 Diseño y proyecto, y CT-09 Pensamiento crítico (Competencias transversales UPV, 2019).

Si bien existen programas informáticos para la realización del diseño o del análisis económico, como es el caso del PVSyst, SAM o HOMER, estos programas tienen un coste elevado y una metodología definida para realizar los cálculos, dicha metodología no se puede modificar. El proceso de diseño de una microrred puede ser laborioso si este se realiza manualmente, una de las herramientas más conocidas y utilizadas por los estudiantes durante sus estudios de grado y master es la hoja de cálculo de MS Excel, que es el recurso tecnológico utilizado en esta metodologí. Se trata de utilizar una herramienta habitual y aplicar los conocimientos al diseño de microrredes para simplificar el proceso, con el 
consecuente ahorro de tiempo y disminución de posibles errores. Por tanto, se mejora la metodología utilizando nuevos recursos informáticos. El uso de MS Excel es muy habitual tanto a nivel profesional, como a nivel educativo. Muchos profesores lo utilizan para realizar prácticas de laboratorio o informáticas. Existen diversas publicaciones en la que profesores utilizan MS Excel para aplicar la metodología de la práctica como es el caso de (AlcázarOrtega, 2018), (Mora, 2010) y (Cuenca, 2010).

\section{Objetivos}

\subsection{Objetivo general}

El objetivo de este trabajo es presentar una metodología que permita a los estudiantes adquirir competencias transversales mediante su aplicación a la práctica 2 de la asignatura Redes Eléctricas Inteligentes: Diseño de una microrred para autoconsumo basada en energías renovables.

\subsection{Objetivos específicos}

- Aplicar la metodología elaborada en la práctica.

- Que el estudiante refuerce sus conocimientos en el uso de recursos tecnológicos para una aplicación práctica, para ello se utilizará como herramienta MS Excel.

- Que el estudiante desarrolle las competencias transversales UPV 1 a 5 y 9.

- Que, con los conocimientos adquiridos, el estudiante se capaz de utilizar la metodología en un ámbito profesional.

\section{Desarrollo de la innovación}

\subsection{Metodología}

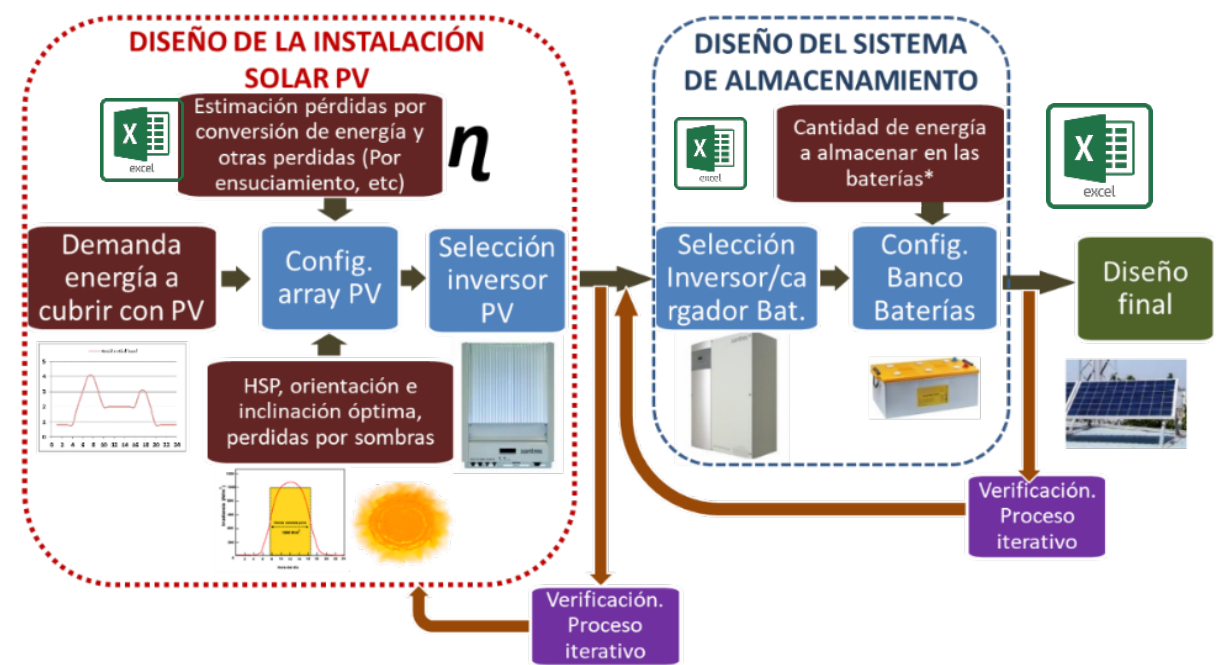

Fig. 1 Proceso de diseño de una microrred para autoconsumo basada en solar PV y almacenamiento en baterías. * Depende de la cantidad de energía que se quiera almacenar.

La metodología para realizar el diseño se aplica al laboratorio de recursos energéticos distribuidos de la UPV (LabDER). El método se puede extrapolar a otras aplicaciones. El proceso de diseño se muestra en la figura 1 . Se observan en marrón los inputs que se requieren 
para el diseño, en azul los outputs obtenidos a partir de los inputs, que luego pasan a ser inputs del siguiente paso. Con el fin de que no se sobrepasen los valores máximos de los equipos seleccionados, después de hacer los cálculos y diseñar cada subsistema, este se verifica mediante la comprobación de tensiones y corriente de entrada a los dispositivos de conversión (Inversor de conexión a red e inversor cargador). Este paso se realiza mediante un proceso iterativo como se indica en la figura 1.

\subsection{Inputs}

Como se observa en la figura 1, los inputs para la configuración del sistema fotovoltaico son:

- Demanda de energía a cubrir: La demanda de energía a cubrir se obtiene midiendo el consumo de energía del sitio donde se ubicará la instalación. Si no se dispone de esta información o es una obra nueva, se calcularía estimando el consumo total a partir de los futuros equipos consumidores. Para ello se recopilaría la información indicada en la tabla 1.

Tabla 1. Plantilla para estimar el conusumo de energía de una instalación futura.

\begin{tabular}{ccccc}
\hline Equipo & Unidades & Potencia & Horas de uso al mes & Consumo total (kWh) \\
\hline Iluminación & 20 & 30 & 100 & 60 \\
.. &.. &.. &.$\cdot$ &.. \\
\hline Total &.. &.$\cdot$ &.$\cdot$ & Total consumo/mes \\
\hline
\end{tabular}

La potencia máxima se obtiene estimando a qué horas del día consumen energía cada uno de los equipos y sumando los valores horarios hasta obtener una curva de demanda diaria. Se determinaría de esta manera la potencia máxima de todo el día. En este tipo de casos, donde la obra es nueva, se tendría una curva de carga con una incertidumbre elevada, ya que el consumo real suele diferir del estimado.

Tabla 2. Demanda real de Energía del LabDER

\begin{tabular}{lc}
\hline Consumo anual & 8929 \\
Día de mayor consumo del año $(\mathrm{kWh})$ & 32 \\
P instantánea máx. $(\mathrm{kW})$ & 12 \\
\hline
\end{tabular}

En caso de disponer de la demanda real (si se tiene un analizador de redes eléctricas), la incertidumbre baja, ya que se estimaría la demanda futura con valores reales del pasado. Si se prevén nuevas cargas están deben ser tenidas en cuenta en la curva de demanda. En la tabla 2 se muestran la demanda real de energía del LabDER, que es el sitio donde se ubicaría la instalación diseñada

- Horas de sol pico (HSP) u horas de sol equivalentes: son las horas al día que la irradiación ha sido equivalente a $1000 \mathrm{~W} / \mathrm{m}^{2}$. Dependiendo de la ubicación, en España el recurso solar va desde 1200 hasta 2000 HSP/año en el plano horizontal y desde 1300 hasta $2200 \mathrm{HSP} /$ año si el panel se instala con el ángulo óptimo (PVGIS, 2019). Para calcular las HSP en una ubicación específica se recurre a bases de datos. Una de las bases de datos más utilizada en Europa es PVGIS (http://re.jrc.ec.europa.eu/pvg_tools/en/tools.html), la cual pertenece a la comisión 
europea y se puede consultar de forma gratuita mediante su página web. PVGIS incluye 4 fuentes de datos distintas. Además también se podría utilizar la base de datos de la nasa (https://power.larc.nasa.gov/data-access-viewer/).

\begin{tabular}{|c|c|c|c|c|c|}
\hline GRID CONNECTED & $\frac{109}{109}$ MONTHIY IRRADIATII & & & Provided inputs: & \\
\hline TRACKNG PV & Solar radiation database $e^{2}$ & PVGIS-CI & . & Location [Lat/Lon]: & $39.483,-0.338$ \\
\hline OFF-GRID & Start year: $\quad 2014 \quad \checkmark$ & End year: & $2016 \quad \checkmark$ & Horizon: & Calculated \\
\hline & $\begin{array}{l}\text { Irradiation: } \\
\square \text { Global horizontal irradiation }\end{array}$ & & & Database used: & PVGIS-CMSAF \\
\hline DAILY DATA & $\begin{array}{l}\square \text { Direct normal irradiation } \\
\checkmark \text { Global irradiation optimum angle }\end{array}$ & & & Simulation outputs: & \\
\hline HOURLY DATA & $\begin{array}{l}\text { Global irradiation at angle. } \\
\text { Ratio: }\end{array}$ & $(0-90)$ & & Slope angle $\left[^{\circ}\right]$ : & 36 (opt) \\
\hline TWY & $\begin{array}{l}\text { Diffuse/global ratio } \\
\text { Temperature: }\end{array}$ & & & Azimuth angle $\left[{ }^{\circ}\right]$ : & 0 \\
\hline
\end{tabular}

Fig. 2 Inputs utilizados para obtener las HSP de la web de PVGIS e inclinación obtenida

La figura 2 muestra los datos de entrada utilizados para obtener las HSP a través del PVGIS. Las coordenadas del LabDER son: 39.483, -0.338. La fuente de datos seleccionada es CMSAF. Como input del diseño se ha utilizado la media de los últimos 3 años. La tabla 3 muestra los resultados obtenidos del programa (HSP a la inclinación optima en la ubicación donde se encuentra el LabDER). La inclinación óptima es calculada por PVGIS y es de $36^{\circ}$ (Figura 2). Como resultado se obtiene que la media de los años 2014 a 2016 es de 2022 HSP al año (2036 HSP en 2014 , 2017 HSP en 2018 y 2002 HSP en 2016).

Tabla 3. HSP en la ubicación del LabDER de 2012 a 2016 obtenidas de PVGIS

\begin{tabular}{lcccc}
\hline Month/Year & $\mathbf{2 0 1 4}$ & $\mathbf{2 0 1 5}$ & $\mathbf{2 0 1 6}$ & Promedio \\
\hline Jan & 106 & 141 & 104 & 117 \\
Feb & 119 & 125 & 139 & 128 \\
Mar & 182 & 167 & 179 & 176 \\
Apr & 195 & 197 & 185 & 192 \\
May & 208 & 218 & 201 & 209 \\
Jun & 202 & 210 & 213 & 208 \\
Jul & 216 & 211 & 213 & 213 \\
Aug & 214 & 203 & 213 & 210 \\
Sep & 178 & 164 & 193 & 178 \\
Oct & 174 & 132 & 145 & 150 \\
Nov & 114 & 138 & 111 & 121 \\
Dec & 128 & 121 & 106 & 118 \\
\hline Total & 2036 & 2027 & 2002 & $\mathbf{2 0 2 2}$ \\
\hline
\end{tabular}

Fuente: (PVGIS, 2019)

- Estimación de pérdidas: Entre las pérdidas a tener en cuenta se encuentran:

- Pérdidas por conversión de energía en los equipos. Para iniciar el cálculo se pueden utilizar los siguientes valores: inversor $(\eta=90 \%)$, cargador $(\eta=85 \%)$ y cableado (Pérdidas $=1 \%)$. Una vez seleccionados los equipos se podría utilizar la eficiencia dada por el fabricante del equipo seleccionado. La figura 3 muestra los cálculos realizados para estimar la cantidad de energía que se debe generar teniendo en cuenta las perdidas. 
De la tabla 3 se obtiene la demanda máxima diaria que es de $32 \mathrm{kWh}$, sin embargo, debido a las pérdidas la energía, los paneles deben generar 42,3 $\mathrm{kWh}$ para cubrir dicha demanda.

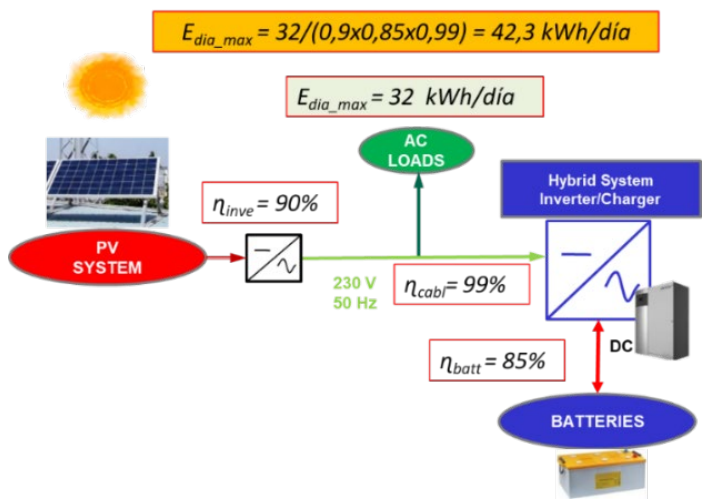

Fig. 3 Cálculo de la energía máxima demanda teniendo en cuenta las pérdidas por conversión de energía.

- Otras pérdidas: Aquí se tienen en cuenta el resto de pérdidas. Probablemente las mayores sean las perdidas por trabajar a una temperatura diferente a la temperatura de operación de la célula solar. Como se observa en la figura 9 para esta pérdida se utilizará un valor del $10 \%$. Se utilizará además un valor del $3.5 \%$ para las pérdidas por ensuciamiento.

\subsection{Outputs}

\subsubsection{Selección de los paneles}

Con la información de los inputs se dimensiona el array de paneles fotovoltaicos y se determina el tipo y la cantidad a utilizar. Existen varios criterios de selección, como pueden ser: la marca, la calidad, el país de procedencia, etc. Para simplificar el cálculo y por ser un criterio utilizado frecuentemente, se trabajará con el panel europeo que mejor precio tenga por kWp. Para ello se utilizará un presupuesto solicitado a cuatro empresas en el año 2017. Con este presupuesto se ha elaborado la tabla 4 . Los paneles seleccionados son de $270 \mathrm{~W}_{\mathrm{p}}$ de la marca RED, ofertados por la empresa 2. Hay paneles con un mejor precio fabricados en china, pero una de las condiciones impuestas es que el panel fuese de fabricación europea. Las características del panel seleccionado se muestran en la figura 4.

\begin{tabular}{|l|l|}
\hline Modelo & RED270-60 M \\
\hline Potencia máxima (Pmax) & $270 \mathrm{~W}$ \\
\hline Tensión de potencia máx. (Nmp) & $31,48 \mathrm{~V}$ \\
\hline Tensión de corriente máx. (Imp) & $8,58 \mathrm{~A}$ \\
\hline Tensión de circuito abierto (Voc) & $38,56 \mathrm{~V}$ \\
\hline Corriente de cortocircuito (Isc) & $9,27 \mathrm{~A}$ \\
\hline Eficiencia de célula (\%) & $19.40 \%$ \\
\hline Máxima tensión del sistema (M) & $1000 \mathrm{VDC}$ \\
\hline Coef. de temp Isc (\%) ${ }^{\circ} \mathrm{C}$ & $0,037 \% /{ }^{\circ} \mathrm{C}$ \\
\hline Coef. de temp Voc (\%) ${ }^{\circ} \mathrm{C}$ & $-0,34 \% /{ }^{\circ} \mathrm{C}$ \\
\hline Coef. de temp Pmax (\%) ${ }^{\circ} \mathrm{C}$ & $-0,48 \% /{ }^{\circ} \mathrm{C}$ \\
\hline $\begin{array}{l}\text { Temperatura nominal } \\
\text { de funcionamiento de célula }\end{array}$ & $45 \pm 2^{\circ} \mathrm{C}$ \\
\hline
\end{tabular}

Fig. 4 Especificaciones técnicas del panel solar fotovoltaico seleccionado. 
Tabla 4. $€ / \mathrm{kW}$ de los paneles presupestados a cuatro empresas distintas.

\begin{tabular}{llrrr}
\hline \multicolumn{1}{c}{ Paneles solares } & Pot $(\mathbf{W})$ & Coste & $\boldsymbol{\epsilon} / \mathbf{k W}$ \\
\hline $1 \quad$ Green Power 170 w monocristalino & 169,8 & $194,0 €$ & 1,14 \\
& Atersa A-75 P & 75,1 & $157,0 €$ & 2,09 \\
Atersa A-200 M & 200,0 & $277,0 €$ & 1,39 \\
Atersa A-240 P & 239,8 & $218,0 €$ & 0,91 \\
A- 250 P GSE & 250,7 & $231,0 €$ & 0,92 \\
Atersa A-240 P & 239,8 & $218,0 €$ & 0,91 \\
A- 310 P GSE & 313,1 & $261,0 €$ & 0,83 \\
185W Mono PLUTO185/Ade - SUNTECH & 169,8 & $147,7 €$ & 0,87 \\
250W Mono - STP250S-20/Wd - SUNTECH & 250,7 & $217,8 €$ & 0,87 \\
320W poli - KD320GH-4YB - KYOCERA & 313,1 & $393,3 €$ & 1,26 \\
RED270-60M-270W (Efi=19,40\%) & $\mathbf{2 7 0 , 1}$ & $\mathbf{2 2 2 , 8 €}$ & $\mathbf{0 , 8 3}$ \\
JinKo JKM200M-72 (EU) 200W & 200,0 & $152,8 €$ & 0,76 \\
AMERISOLAR AS-5M 210W (Ef=16,45\%) & 210,4 & $186,30 €$ & 0,89 \\
MÓDULO FV LUXOR LX200M 200 Wp & 201,5 & $182,0 €$ & 0,90 \\
MÓDULO FV AXITEC AC250P 250 Wp & 251,1 & $198,0 €$ & 0,79 \\
MÓDULO FV AXITEC AC300P 300 Wp & 300,5 & $242,0 €$ & 0,81 \\
LUXOR Ecoline 72/200 Wp & 201,5 & $203,0 €$ & 1,01 \\
LUXOR Ecoline 60/250 Wp & 251,6 & $223,0 €$ & 0,89 \\
Axiworld power AC-300P/156-72s(MIA) & 300,5 & $270,0 €$ & 0,90 \\
\hline
\end{tabular}

\subsubsection{Selección del inversor de conexión a red}

Utilizando el criterio de menor coste por $\mathrm{kW}$ instalado usado en los paneles solares, se selecciona un inversor de red de fabricación europea. Como suele ocurrir con este tipo de dispositivos, los inversores más grandes tendrán un menor coste por $\mathrm{kW}$, por tanto, hay que comparar entre inversores del mismo orden de tamaño y siempre de acuerdo con la potencia solar estimada calculada anteriormente. La potencia máxima producida por los paneles solares es cercana a los $9 \mathrm{~kW}$, por tanto, se requieren tres inversores con una potencia mínima de entrada de $3 \mathrm{~kW}$ (red trifásica). Se ha escogido el inversor Schneider RL3000 de $3 \mathbf{~ k W}$ como se muestra en la tabla 5. Las características del inversor se muestran en la figura 5.

\begin{tabular}{|c|c|c|c|}
\hline Device short name & RL $3000 \mathrm{E}$ & Output (AC) & \\
\hline Electrical specifications & & Nominal output power & $3 \mathrm{kVA}$ \\
\hline Input (DC) & & Nominal output voltage & $230 \mathrm{~V}$, single-phase \\
\hline MPPT voltage range, full power & $160-500 \mathrm{~V}$ & Isolation & Transformerless \\
\hline Operating voltage range & $90-550 \mathrm{~V}$ & AC voltage range & $184 V-276 V$ \\
\hline Starting voltage & $100 \mathrm{~V}$ & Frequency & $50 / 60 \mathrm{~Hz}$ \\
\hline Max. input voltage, open circuit & $550 \mathrm{~V}$ & Frequency range & $50 / 60 \mathrm{~Hz}+/-5 \mathrm{~Hz}$ \\
\hline Number of MPPT & 2 & Max. output current & $13.9 \mathrm{~A}$ \\
\hline Max. input current per MPPT & $10 \mathrm{~A}$ & Total harmonic distortion & $<3 \%$ \\
\hline Max. short circuit current per MPPT & $13.9 \mathrm{~A}$ & Power factor (adjustable) & 0.8 lead to $0.8 \mathrm{lag}$ \\
\hline Nominal input power for max. output & $3.2 \mathrm{~kW}$ & AC connection type & IP67 connector \\
\hline Max. DC input power per MPPT & $3.2 \mathrm{~kW}$ & Efficiency & \\
\hline DC connection type & MC4, 2 pairs $(1+1)$ & Peak & $97.5 \%$ \\
\hline DC switch & Integrated (optional) & European & $97.0 \%$ \\
\hline
\end{tabular}

Fig. 5 Especificaciones técnicas del inversor de conexión a red seleccionado.

Tabla 5. $€ / \mathrm{kW}$ para los inversores de red presupuestados en cuatro empresas distintas. 
Diseño de un sistema híbrido basado en energía solar PV con almacenamiento en baterías: aplicación a la enseñanza de Microrredes Eléctricas utilizando Microsoft Excel

\begin{tabular}{llrrr}
\hline \multicolumn{1}{c}{ Inversor } & Pot $(\mathrm{W})$ & Precio con IVA & $€ / \mathrm{Wp}$ \\
\hline 1 & Ingecon Sun Lite 3 TL de 3 KW & 3000 & $1614 €$ & 0,54 \\
& Invesor monofásico Kostal piko 3 & 3000 & $1131 €$ & 0,38 \\
\multirow{2}{*}{2} & Invesor monofásico Kostal piko 3.6 & 3600 & $1251 €$ & 0,35 \\
& Invesor monofásico Kostal piko 3 & 3000 & $826 €$ & 0,28 \\
& Invesor monofásico Kostal piko 3,6 & 3600 & $979 €$ & 0,27 \\
& Inversor Schneider RL3000 (3 kW) & $\mathbf{3 0 0 0}$ & $\mathbf{8 7 3 €}$ & $\mathbf{0 , 2 9}$ \\
& Inversor Schneider RL4000 (4 kW) & 4000 & $1034 €$ & 0,26 \\
\multirow{3}{*}{3} & Inversor Schneider RL5000 (5 kW) & 5000 & $1083 €$ & 0,22 \\
& Inversor Schneider RL3000 (3 kW) & 3000 & $1011 €$ & 0,34 \\
& Inversor Schneider RL4000 (4 kW) & 4000 & $1302 €$ & 0,33 \\
& Inversor Schneider RL5000 (5 kW) & 5000 & $1585 €$ & 0,32 \\
& Invesor monofásico Kostal piko 3 & 3000 & $1076 €$ & 0,36 \\
& Invesor monofásico Kostal piko 3.6 & 3600 & $1246 €$ & 0,35 \\
Invesor monofásico Kostal piko 3 & 3000 & $1075 €$ & 0,36 \\
& Invesor monofásico Kostal piko 3.6 & 3600 & $1246 €$ & 0,35 \\
Inversor Schneider RL3000 (3 kW) & 3000 & $1041 €$ & 0,35 \\
\hline
\end{tabular}

\subsubsection{Selección del inversor/cargador}

Siguiendo en la misma línea de la selección de los paneles solares y del inversor de conexión a red, se escoge el inversor/cargador europeo, con el mejor coste por $\mathrm{kW}$, en este caso sería el XW+7048E de la marca SCHNEIDER de 4,5 KW. Se requieren 3 para producir los $12 \mathrm{~kW}$ de demanda máxima. Los inversores presupuestados se muestran en la tabla 5. En la figura 6 se muestran las características del inversor/cargador seleccionado.

Tabla 5. $€ / \mathrm{kW}$ para los inversores/cargadores (calculado del presuspuestos solicitados).

\begin{tabular}{llrrr}
\hline & \multicolumn{1}{c}{ Inversor/ cargador } & Potencia $(\mathrm{W})$ & Precio $(€)$ & $€ / \mathrm{Wp}$ \\
\hline 1 & Inversor SMA mod. SB3000 TL de $3 \mathrm{Kw}$ & 3200 & $1614 €$ & 0,50 \\
& Inversor cargador híbrido XW4500 w 48v & 4500 & $2698 €$ & 0,60 \\
& Inversor cargador híbrido XW6000 w 48 v & 6500 & $2998 €$ & 0,46 \\
2 & Inversor cargador Victron 48/5000/70-50 A & 5000 & $2220 €$ & 0,44 \\
& Inv/carg. 4500W-48V-230VAC XW4548E & 4500 & $2270 €$ & 0,50 \\
& Inv/carg. 6000W-48V-230VAC XW6048E & 6000 & $2524 €$ & 0,42 \\
& Conext XW+7048E de SCHNEIDER 5500W & $\mathbf{6 ~ 8 0 0}$ & $\mathbf{3 3 2 5}$ & $\mathbf{0 , 4 9}$ \\
& Conext XW+8548E de SCHNEIDER 6800W & 6800 & $3882 €$ & 0,57 \\
3 & Inversor cargador híbrido XW4500 w 48v & 4500 & $2570 €$ & 0,57 \\
& Inversor cargador híbrido XW6000 w 48 v & 6000 & $2867 €$ & 0,48 \\
Victron multiplus 48/5000/700-100 & 5000 & $2536 €$ & 0,51 \\
& Inversor cargador híbrido XW4500 w 48v & 4500 & $2570 €$ & 0,57 \\
Inversor cargador híbrido XW6000 w 48 v & 6500 & $2968 €$ & 0,46 \\
\hline
\end{tabular}

\subsubsection{Selección de las baterías}

La selección de las baterías, si se tiene en cuenta la rentabilidad, es más compleja que el resto de dispositivos, ya que la duración de las mismas y la profundidad de descarga (DoD) cambia significativamente en función de la tecnología. Actualmente el uso de baterías de Iones de 
Litio está tomando fuerza en instalaciones renovables. En esta publicación, debido a su coste y a que son las más utilizadas en la actualidad, se realizarán los cálculos con baterías de ácido plomo, pero podría utilizarse el mismo procedimiento para seleccionar baterías de Iones de litio. La batería seleccionada es las Monoblock Saclima de 250 A y 12 V (Tabla 7) y las características se muestran en la figura 7.

\begin{tabular}{|c|c|c|c|}
\hline Device short name & $X W+7048 E$ & Charger DC output & \\
\hline Inverter AC output & & Maximum output charge current & $110 \mathrm{~A}$ \\
\hline Output power (continuous) at $25^{\circ} \mathrm{C}$ & $5500 \mathrm{~W}$ & Output charge voltage range & $40-64 \mathrm{~V}$ ( 48 V Nominal) \\
\hline Overload $30 \mathrm{~min} / 60 \mathrm{sec}$ at $25^{\circ} \mathrm{C}$ & $7000 \mathrm{~W} / 9500 \mathrm{~W}$ & Charge control & Three stage, two stage, boost, custom \\
\hline Output power (continuous) at $40^{\circ} \mathrm{C}$ & $4500 \mathrm{~W}$ & Charge temperature compensation & Battery temperature sensor included \\
\hline & & Power factor corrected charging & 0.98 \\
\hline 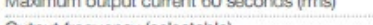 & $40 \mathrm{~A}$ & Competible battery types & Flooded (delautt), Gel, AGM, LON, custom* \\
\hline Output frequency (selectable) & $50 / 60 \mathrm{~Hz}$ & Battery bank range (scaled to PV array size) & 440 to $10000 \mathrm{Ah}$ \\
\hline Output voltage & $230 \mathrm{Vac}$ & AC input & \\
\hline Total harmonic distortion (THD) at rated power & $<5 \%$ & AC 1 (gridi) input current (selectable limit) & $3.60 \mathrm{~A}(56 \mathrm{~A}$ default) \\
\hline Idle consumption search mode & $<7 W$ & AC 2 (generatort) input current (seloctable imit) & $3.60 \mathrm{~A}$ (56 A detault) \\
\hline Input DC voltage range & 40 to $64 \mathrm{~V}$ (48 V Nominal) & AC inpus yoltace nominal & $230 \mathrm{~V}+1 \cdot 3 \%$ \\
\hline Maximum input DC current & $150 \mathrm{~A}$ & AC input frequency range (bypass/charge mode) & $45-55 \mathrm{~Hz}$ (detault) $40-68 \mathrm{~Hz}$ (allowable) \\
\hline
\end{tabular}

Fig. 6 Especificaciones técnicas del inversor/cargador seleccionado.

Tabla 7. €/kW para las baterias (calculado del presuspuestos solicitados).

\begin{tabular}{llccc}
\hline & \multicolumn{1}{c}{ Baterías } & Pot $(\mathbf{W})$ & Precio $(\boldsymbol{\epsilon})$ & $\boldsymbol{\epsilon} / \mathbf{W p}$ \\
\hline 1 & Enersol 250 Ah $-12 \mathrm{v}$ & 3000 & 375 & 0,13 \\
2 & Saclima 220A (C20) - 250A (C100) & $\mathbf{3 0 0 0}$ & $\mathbf{2 0 0}$ & $\mathbf{0 , 0 7}$ \\
3 & Enersol 250 Ah $-12 \mathrm{v}$ & 3000 & 235 & 0,08 \\
4 & Enersol 250 Ah $-12 \mathrm{v}$ & 3000 & 350 & 0,12 \\
\hline
\end{tabular}

\begin{tabular}{|c|c|}
\hline Voltaje & $12 \mathrm{v}$ \\
\hline C10OAh & $250 \mathrm{AH}$ \\
\hline C2OAh & $230 \mathrm{AH}$ \\
\hline Peso & $60,70 \mathrm{~kg}$ \\
\hline Ancho & $274 \mathrm{~cm}$ \\
\hline Largo & $518 \mathrm{~cm}$ \\
\hline Alto & $242 \mathrm{~cm}$ \\
\hline
\end{tabular}

Fig. 7 Especificaciones técnicas las baterías seleccionadas.

\subsection{Cálculos}

Todos los cálculos son realizados por el estudiante, por tanto, a diferencia de una aplicación informática, el estudiante puede analizar, modificar o mejorar las ecuaciones utilizadas para realizar los cálculos. En el diseño realizado se utilizan las ecuaciones 1 y 2 para determinar el número de paneles:

$P_{\text {pic_min }}=\frac{\text { Demanda max diaria energía }\left(\frac{k W h}{d i a}\right)}{H S P / \text { dia }}$

$N_{\text {paneles }}=\frac{P_{\text {pic_min }}}{P_{\text {pic_panel }}}$

La cantidad de inversores se calcula con las ecuaciones 3 y 4 :

$P_{\max \_M P P_{1}}(k W)=P_{\text {pic_panel }} \cdot N_{\text {paneles }}$ 
$N_{\text {inv }}=\frac{P_{\max \_M P P}}{P_{\text {max } \_ \text {in } \_D C}}$

Donde $P_{\text {max }}$ in_DC es la potencia máxima en DC que soporta el inversor (Proveniente de los paneles solares). La cantidad de inversores/cargadores se calcula con la ecuación 5:

$N_{\text {inv }}=\frac{P_{\text {max_inst }}}{P_{\text {nom_inv }}}$

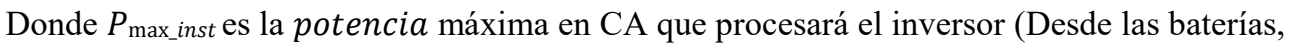
la instalación PV o a hacia las cargas)

El número de baterías se calcula con las ecuaciones 6 y 7 (Smets, 2016). Primero se debe calcular la capacidad mínima de almacenamiento requerida y luego el número de baterías. Para evitar las perdidas por transporte de energía, se trabajará a la tensión de baterías más alta posible. Los inversores cargadores comerciales normalmente pueden trabajar a 12, 24 y $48 \mathrm{~V}$. Algunos trabajan a $60 \mathrm{~V}$, pero en el inversor seleccionado la máxima tensión del banco de baterías soportada es de $48 \mathrm{~V}$, por ello se utilizarán $48 \mathrm{~V}$.

$$
\text { Mínimo } C_{\text {Batt }}=\frac{E_{\text {req }}}{D o D \cdot V_{\text {charg }}}=\frac{E_{\text {dia }} \cdot D O A}{D o D \cdot V_{\text {charg }}}
$$

Donde DOA es el número de días de autonomía y $D o D$ es la profundidad de descarga que recomienda el fabricante para esa batería. El número de bancos de baterías (Un banco tiene 4 baterías de $12 \mathrm{~V}$ en serie para llegar a los $48 \mathrm{~V}$ requeridos por el inversor/cargador) se calcula con la ecuación 7 .

$$
N_{\text {Banc_Batt }}=\frac{\text { Mínimo } C_{\text {Batt }}}{\text { Batt }_{\text {capacity }}}
$$

El número de baterías totales sería igual al número de bancos multiplicado por el número de baterías de cada banco (que en este caso es de 4).

\section{Resultados - Utilización de MS Excel}

\subsection{Inputs}

Para realizar los cálculos de forma adecuada, llevar a cabo nuevos diseños y comparar entre distintas marcas se utiliza Microsoft Excel (se pueden utilizar más columnas para comparar distintos diseños). Los valores de entrada del diseño se muestran en la figura 8. Los datos de entrada principales son obtenidos de la curva de carga (Consumo y potencia máxima), eficiencia de los equipos de conversión, recurso solar y la disminución de dicho recurso debido a las pérdidas.

\subsection{Configuración del array fotovoltaico}

El número de paneles que se deben utilizar se determinan a partir del diseño del array que se muestra en la figura 9. Como resultado so obtiene que se requieren 33 paneles de $270 \mathrm{Wp}$, por tanto, la instalación solar tendrá una potencia pico de $8,9 \mathrm{~kW}$. Dicha potencia pico no corresponde con la potencia máxima demandada por las cargas. Los paneles solares no suelen producir la potencia requerida en cada momento, por tanto, esta puede ser mayor y el 
excedente se envía a baterías o puede ser menor y por tanto se utiliza energía almacenada en las baterías para alcanzar la potencia demandada, como ocurre por la noche.

\begin{tabular}{|c|c|c|}
\hline 1 & \multicolumn{2}{|l|}{ Cálculos - Stand-alone PV System } \\
\hline 2 & & \\
\hline 3 & Datos de entrada & \\
\hline 4 & Datos de la curva de carga & \\
\hline 5 & Día de máximo consumo del año ( $\mathrm{kWh} /$ día) & 32,0 \\
\hline 6 & Pact máx. (KW) & 12,1 \\
\hline 7 & & \\
\hline 8 & Eficiencia del sistema & \\
\hline 9 & Inversor & $90 \%$ \\
\hline 10 & Cargador & $85 \%$ \\
\hline 11 & Cableado & $99 \%$ \\
\hline 12 & Eficiencia total & $76 \%$ \\
\hline 13 & & \\
\hline 14 & Demanda de energía & \\
\hline 15 & Energía demandada por los paneles el diá de mayor consumo (teniendo en cuenta las pérdidas) ( $\mathrm{kW} / \mathrm{dia}$ ) & 42,3 \\
\hline 16 & & \\
\hline 17 & Horas de sol pico & \\
\hline 18 & HSP (h/año) & 2022 \\
\hline 19 & HSP $(h /$ día) & 5,54 \\
\hline 20 & Pérdidas por sombras (\%) & $\underline{0,0 \%}$ \\
\hline 21 & Pérdidas por ensuciamiento & $3,50 \%$ \\
\hline 22 & Pérdidas por trabajar a una temperatura diferente a la de operación nominal de la célula (TONC) & $10,0 \%$ \\
\hline 23[ & HSP disponobles (descontando sombras) (h/día) & 4,79 \\
\hline
\end{tabular}

Fig. 8 Inputs requeridos para el diseño.

\begin{tabular}{|c|c|c|}
\hline \multicolumn{3}{|c|}{$1 \quad$ Cálculos - Stand-alone PV System } \\
\hline 23 & 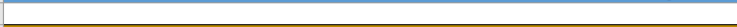 & \\
\hline 24 & Diseño del array (Paneles solares PV) & \\
\hline 25 & Datos paneles solares (Fabricante) & \\
\hline 26 & Marca & RED \\
\hline 27 & Modelo & RED270-60M-270W \\
\hline 28 & Tipo & Mono \\
\hline 29 & Células & 60 \\
\hline 30 & Área células $(\mathrm{cm} 2)$ & 243,36 \\
\hline 31 & n_célula (\%) & $18,5 \%$ \\
\hline 32 & Vmpp (V) & 31,48 \\
\hline 33 & $\operatorname{Impp}(\mathrm{A})$ & 8,58 \\
\hline 34 & P pico $(W p)$ & $\underline{270}$ \\
\hline 35 & $\operatorname{Voc}(\mathrm{V})$ & 38,56 \\
\hline 36 & $\operatorname{Isc}(\mathrm{A})$ & 9,27 \\
\hline 37 & Longitud panel (mm) & 1.640 \\
\hline 38 & Ancho panel (mm) & 992 \\
\hline 39 & Área panel (m2) & 1,627 \\
\hline 40 & n_panel (P_pico_W/(Área_panel_m2 x 1000_W/m2)) & $16,60 \%$ \\
\hline 41 & Datos del array & \\
\hline 42 & Potencia PV pico minima a instalar (Energía dia de mayor consumo/HSP) (Wp) & 8,89 \\
\hline 43 & $\mathrm{~N}$ paneles & 32,90 \\
\hline 44 & N paneles (redondeando) & $\underline{33,00}$ \\
\hline 45 & Ppico total real (W) & $8.913,2$ \\
\hline
\end{tabular}

Fig 9. Resultados de cálculo del número de paneles.

\subsection{Dimensionamiento de los inversores de conexión a red.}

En la figura 10 se muestran el resultado de los cálculos del dimensionado del inversor. Se deduce que se requieren 3 inversores de conexión a red de $3 \mathrm{KW}$. Aunque la potencia instalada de los inversores sea de $8,9 \mathrm{~kW}$, la potencia producida la impone principalmente la radiación solar y la potencia del sistema fotovoltaico. Además, también se muestran los resultados de verificación del inversor, en este caso el voltaje, la intensidad y la potencia de entrada nunca sobrepasan los valores que soporta el inversor.

\subsubsection{Verificación de los inversores de conexión a red}

Una vez realizado los cálculos se verifica que la salida de los paneles cumpla los límites de intensidad y voltaje de entrada admitidos por el inversor. En el ejemplo realizado, 
corresponden 11 paneles a cada inversor. Se utilizan el número máximo de paneles en serie hasta llegar a la tensión máxima permitida en el MPP. Instalando los paneles en serie, la tensión máxima que entregan los paneles es de $31,5 \mathrm{~V}$ y la máxima permitida es de $500 \mathrm{~V}$, por tanto, se cumple el criterio. Análogamente se realiza la misma comprobación para la tensión en vacío, la corriente en el MPP y la corriente de cortocircuito. Los resultados se muestran en la tabla 8 . Se observa que los valores máximos entregados por los paneles son menores que los soportados por el inversor.

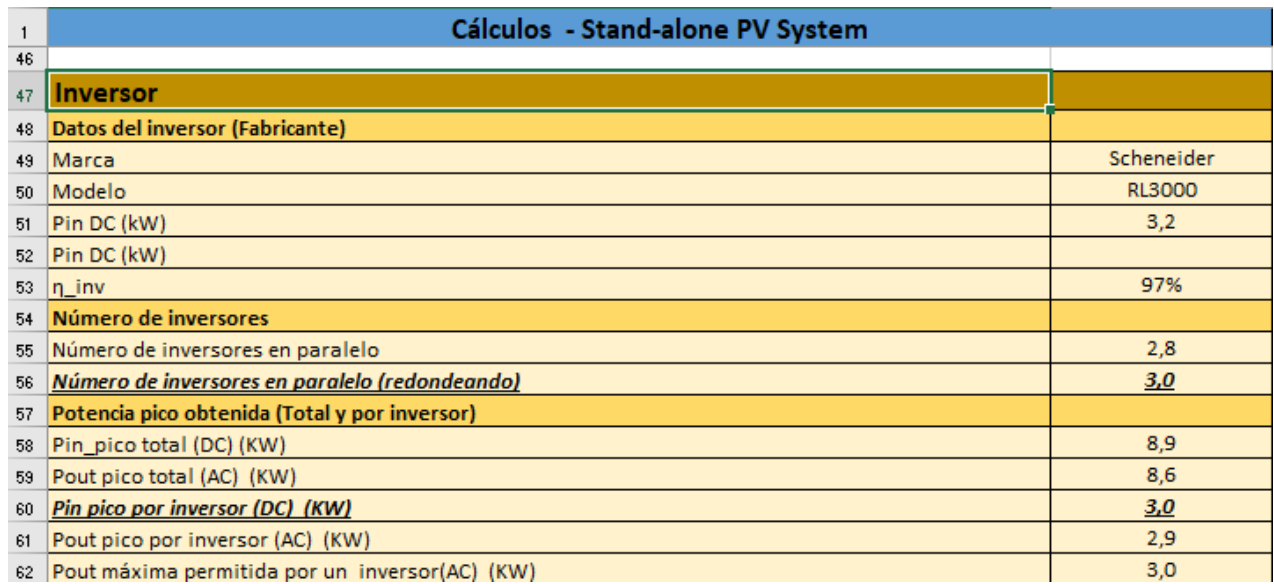

Fig 10. Resultados de cálculo del número de inversores.

Tabla 8. Cálculos para verificar que la salida de los paneles cumpla los criterios del inversor.

\begin{tabular}{lrr}
\hline & \multicolumn{1}{c}{ Max Output paneles } & Max Input inversor \\
\hline Max. input voltage, open circuit & $38,6 \mathrm{~V}$ x 11,0=424,2 V & $550,0 \mathrm{~V}$ \\
Max. short circuit current per MPPT & $9,3 \mathrm{~A}$ & $13,9 \mathrm{~A}$ \\
Operating voltage range & $31,5 \mathrm{~V}$ x 11,0=346,3 V & $500,0 \mathrm{~V}$ \\
Max. input current per MPPT & $9,6 \mathrm{~A}$ & $10,0 \mathrm{~A}$ \\
Nominal input power & $346,3 \times 9,6=3,0 \mathrm{~kW}$ & $3,2 \mathrm{~kW}$ \\
\hline
\end{tabular}

\subsection{Dimensionamiento de los inversores/cargadores.}

En la figura 11 se observan los resultados de los cálculos. En este diseño se utilizarán 3 inversores cargadores de 4,5 kW. Las cargas están conectadas al inversor/cargador, por tanto, será este quien imponga la potencia máxima a entregar que en este caso será de $13,5 \mathrm{~kW}$. La cantidad de energía a entregar por el inversor dependerá de la radiación solar y del estado de carga de las baterías.

\begin{tabular}{|c|c|c|}
\hline \multirow{2}{*}{$\frac{1}{83}$} & \multicolumn{2}{|c|}{ Cálculos - Stand-alone PV System } \\
\hline & & \\
\hline 84 & Inversor cargador & \\
\hline 85 & Datos Inversor cargador & \\
\hline 86 & Marca & Scheneider \\
\hline 87 & Modelo & $X W+7048 E$ \\
\hline 88 & Voltaje operación (12, 24 y $48 \mathrm{~V})$ & 48 \\
\hline 89 & Salida AC del Inversor a cargas (máximos) (Fabricante) & \\
\hline 90 & $\mathrm{P}$ at $25^{\circ} \mathrm{C}$ & 5,5 \\
\hline 91 & $P$ at $40^{\circ} \mathrm{C}$ & 4,5 \\
\hline 92 & Número de equipos & 2,69 \\
\hline 93 & Numero de equipos (redondeando) & $\underline{3,0}$ \\
\hline
\end{tabular}

Fig 11. Resultados de cálculo del número de inversores/cargadores. 


\subsubsection{Verificación del inversor/cargado seleccionado}

De la misma forma que ha verificado el inversor de conexión a red seleccionado, se verifica el inversor/cargador. Debido a que la tensión de entrada es un valor fijo (230 V - Valor eficaz), el único parámetro a verificar es la corriente que llega al inversor/cargador desde el inversor de conexión a red. Este valor es de 13 A (3000W/230V), y se calcula con la potencia máxima entregada por el inversor de conexión a red. El valor soportado por el inversor es 60 A. Por tanto, los inversores cargadores son adecuados.

\subsection{Dimensionamiento del sistema de almacenamiento.}

La figura 12 muestra los cálculos para determinar la cantidad de baterías requeridas. Como resultado se obtiene que se utilizarán 24 baterías de $12 \mathrm{~V}$ y 250 Ah cada una. A cada inversor cargador le corresponden 8 baterías, configuradas en dos bancos en paralelos con 4 baterías en serie cada banco. De esta manera se obtendría la tensión de operación del inversor/cargador que es de $48 \mathrm{~V}$ del lado de las baterías.

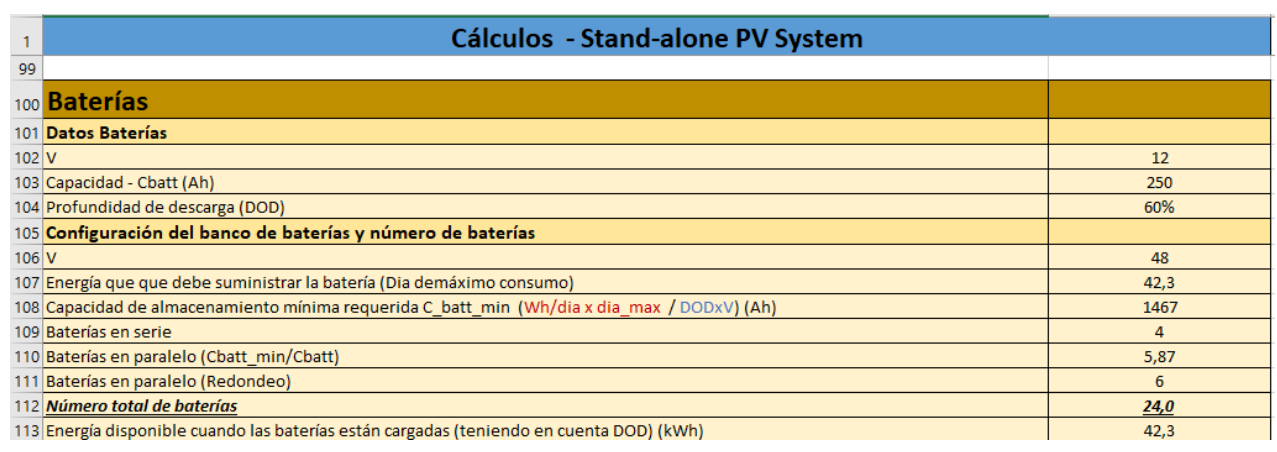

Fig 12. Resultados de cálculo del número de baterías

\subsection{Diseño final}

En la figura 13 se observa el diseño final de la instalación.

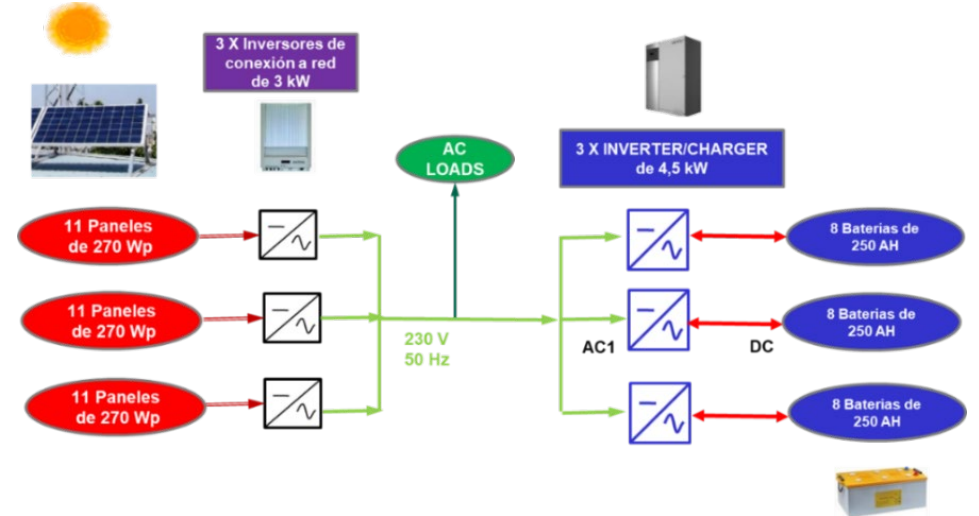

Fig 13. Diseño final de la instalación.

\subsection{Evaluación de la aplicación de la metodología}

Se ha evaluado al estudiante la aplicación de la metodología con una nota ponderando la nota del diseño del sistema solar fotovoltaico y la del sistema de almacenamiento. Los resultados 
Diseño de un sistema híbrido basado en energía solar PV con almacenamiento en baterías: aplicación a la enseñanza de Microrredes Eléctricas utilizando Microsoft Excel

se ven en la figura 13. La evaluación se realizó a un grupo de 15 estudiantes y la nota media fue de 8,8 sobre 10 puntos.

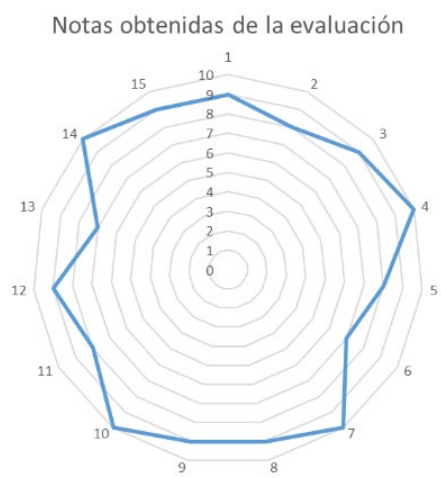

Fig 14. Notas obtenidas de la evaluación de la práctica.

\section{Conclusiones}

El presente artículo presenta una metodología innovadora para diseñar una microrred basada en energía solar fotovoltaica con almacenamiento en baterías mediante la utilización de MS Excel. Una de las ventajas del uso del uso de MS Excel para aplicar la metodología es que simplifica significativamente los cálculos una vez se ha realizado una plantilla. Además, es posible comparar distintos diseños de una manera sencilla.

La nota media obtenida por los estudiantes es de 8,8 puntos sobre 10 . Si bien, debido a su dificultad, no se ha realizado la evaluación de cada una de las competencias transversales, y por tanto no ha analizado el nivel de desarrollo de dichas competencias alcanzado por el estudiante de forma cuantitativa. Esto se realizaría en un trabajo futuro. Sin embargo, en este tipo de métodos, donde se plantea la resolución de un problema real que se podría presentar en un ámbito profesional, el estudiante desarrolla en mayor o menor medida habilidades para la comprensión e integración, aplicación del pensamiento crítico, análisis y resolución de problemas, innovación, creatividad y emprendimiento, diseño de proyecto y pensamiento crítico (CT UPV 1 a 5 y 9).

\section{Agradecimientos}

Este trabajo ha sido respaldado en parte por la administración pública de Valencia bajo la beca ACIF/2018/106.

\section{Referencias}

ALCÁZAR-ORTEGA MANUEL Y ÁLVAREZ-BEL CARLOS. (2018). "Utilización de Microsoft EXCEL en la enseñanza de sistemas eléctricos de potencia: desarrollo de un método matricial para la resolución del problema de despacho económico" En: Congreso Nacional de Innovación Educativa y Docente en Red In-Red. (19 y 20 de julio de 2018, Valencia). Disponible en http://ocs.editorial.upv.es/index.php/INRED/INRED2018/paper/viewFile/8562/3937.

ALEGRE, A., ORTIZ MIRANDA, E.D. y MORENO PÉREZ, O.M. (2012). Lecciones de politica rural: la politica rural de la Unión Europea y su aplicación en España. Valencia: Universitat Politècnica de València. [cuatro o más autores: et al.] 
UNIVERSITAT POLITÈCNICA DE VALÉNCIA. Competencias transversales UPV. < https://www.upv.es/contenidos/COMPTRAN/info/955709normalc.html> [Consulta: Consulta: 14 de Marzo de 2019]

UNIVERSIDAD DE ZARAGOZA. Generación Distribuida, Redes Inteligentes y Movilidad. Máster Universitario en Energías Renovables y Eficiencia Energética< http://titulaciones.unizar.es/guias16/66337_es.pdf> [Consulta: Consulta: 30 de Mayo de 2019]

UNIVERSITAT POLITĖCNICA DE CATALUNYA. Microredes y Optimización Energética. Máster Universitario en Ingeniería en Sistemas Automáticos y Electrónica Industrial < https://www.upc.edu/content/master/guiadocent/pdf/esp/220610 $>$ [Consulta: Consulta: 30 de Mayo de 2019]

UNIVERSIDAD DE VALLADOLID. Microrredes: Un Nuevo Paradigma en el Sistema Energético. Máster en Ingeniería de la Bioenergía y Sostenibilidad Energética< https://alojamientos.uva.es/guia docente/uploads/2018/567/54128/1/Documento.pdf > [Consulta: Consulta: 30 de Mayo de 2019]

UNIVERSIDAD DE ALMERÍA. Microrredes: Gestión Integral de la Energía. Grado en Ingeniería Eléctrica.

http://cms.ual.es/UAL/estudios/grados/plandeestudios/asignaturas/asignatura/GRADO4814 ?idAss $=48143203 \&$ idTit $=4814>$ [Consulta: Consulta: 30 de Mayo de 2019]

CUENCA, D., TAMAYO, R., TAMAYO, J. (2010). Aplicación del Programa Microsoft Excel para resolver problemas experimentales de Física. Sistema de Información Cientifica. Red de Revistas Cientificas de América Latina y el Caribe, España y Portugal (Julio-septiembre, 2010) Centro de Información y Gestión Tecnológica de Santiago de Cuba.

ENERGÍAS RENOVABLES (2019) <https://www.energias-renovables.com/panorama/el-nuevo-realdecreto-ley-15-2018-20181016> y [Consulta: 14 de marzo de 2019]

MORA, R., CÉSPEDES, M. (2010). Excel, Herramienta del Ingeniero. Alicante: Universidad de Alicante.

NATIONAL RENEWABLE ENERGY LABORATORY (NREL). System Advisor Model (SAM). < https://sam.nrel.gov/> [Consulta: Consulta: 14 de Marzo de 2019]

NATIONAL RENEWABLE ENERGY LABORATORY (NREL). HOMER Energy. < https://sam.nrel.gov/> [Consulta: Consulta: 14 de Marzo de 2019]

PHOTOVOLTAIC GEOGRAPHICAL INFORMATION SYSTEM - PGIS. $<$ http://re.jrc.ec.europa.eu/pvg_download/map_index.html\#! > [Consulta: 14 de Marzo de 2019] [Institucional]

SMETS, A., JÄGER, K., ISABELLA, O.,VAN SWAAIJ R. et al (2016) Solar Energy: The physics and engineering of photovoltaic conversion, technologies and systems. UIT Cambridge.

PVSYST PHOTOVOLTAIC SOFTWARE. <https://www.pvsyst.com/> [Consulta: 14 de Marzo de 2019].

ROLDAN PORTA, CARLOS. Guía docente de la asignatura Redes Eléctricas inteligentes, Master universitario en Tecnología Energética para el desarrollo sostenible, Univesitat Politècnica de València. 2018. 